\title{
Glossary of Palaeographical Terms employed in the Text
}

\section{Structure (Italian: 'tratteggio')}

This refers to the number, sequence and direction of strokes which make up each letter. This determines not only the form of the letter but also the possible modifications of it. The dynamic aspect of writing, in other words, the relationship between the number of strokes and the speed of execution (see ductus), affects the structure by encouraging a reduced number of strokes, with a merging of two or more elements (in a single letter or in several letters in succession), a simplification of the structure and a modification of the original form of the letter.

In palaeographical studies in French and English, the Italian term 'tratteggio', here translated as 'structure', corresponds to ductus.

\section{Ductus}

The term ductus here indicates the rate of speed of writing, in other words the dynamic dimension of writing or the time needed to write the signs and, as a result, the effect this has on the final appearance of the script. Seen in absolute terms, it is not possible to quantify the speed of writing since this depends on a variety of concrete factors which are unavailable to us (such as the enviromental and immediate conditions of the act of writing, the scribe's abilities, the materials and instruments which were used, the graphic types adopted, etc.); what it is possible to evaluate is the effect of speed on the final appearance of the writing: the more strokes in single letters or in a succession of letters tend to merge in the smallest possible number of movements the greater the speed of writing. It is usual to distinguish two extremes of speed: set ductus, in other words written with a slow ductus, and cursive ductus, written with a fast ductus.

\section{Writing trace (Italian: 'tracciato')}

The term 'writing trace' refers to the quality of the stroke which defines the design or form of each single letter, in other words the external appearance ('form') they have when they have been written. The writing trace can be thick or thin, uniform and contrasted (according to variations in the thickness of the traces), rounded or angular. The writing trace ('tracciato') must be not be con- 
fused with the structure ('tratteggio'): the latter is a primary structural element, while the former refers to the stylistic characteristic of the written signs. Letters with the same structure can be written with a different writing trace (uniform or contrasted, rounded or angular, etc.). The writing trace also depends on material and dynamic factors.

\section{Module (Italian: 'modulo')}

The term 'module' refers to the shape and relative dimensions of the letters. The shape is determined by the relationship between the height and width of each individual letter, which can be linked to geometric figures: square module $=$ height is equal to width; rectangular module with long side on base line = height is less than width; rectangular module with short side on base line = height is greater than width. The script as a whole is unimodular when all letters are uniform in dimension and shape, and can be inscribed in a square (there is no unimodular script with all letters inscribed in a rectangle); the script is bimodular when there is a contrast between square and rectangular letters: this contrast is often determined by the narrow letters epsilon, theta, omicron and sigma, and the broad letters such as delta, eta, mu, nu, pi and omega.

\section{Writing angle (Italian: 'angolo di scrittura')}

The writing angle measures the position of the writing instrument compared to the base line, since this position determines the thickness of the strokes which make up the letters, the so-called 'chiaroscuro', or shading.

\section{Angle of slope (Italian: 'angolo di inclinazione della scrittura')}

This refers to the angle of inclination of the letters in relation to the base line of the writing: an angle above $90^{\circ}$ means the writing inclines to the right of the axis of the letters, whereas below $90^{\circ}$ (a fairly rare occurrence) means that it inclines to the left of the axis. This parameter can be useful when applied to the analysis of formal scripts (styles or canons) in which, as a distinguishing characteristic, there is an inclination of the axis of the letters (e.g. sloping pointed majuscule). 


\section{Stylistic class (Italian: 'classe stilistica')}

A 'stylistic class' is a set of writings sharing a general framework, form and structure (i.e. the number, sequence and direction of strokes) of some (but not necessarily all) letters; moreover, they may contain graphic variants of the same letter. The term 'stylistic class' attempts to recognise a distinctive writing which does not follow rigid, fixed rules.

\section{Style (Italian: 'stile')}

Individual 'styles' are formed within a 'stylistic class', when the most frequent and most typical characteristics are selected and organized in a graphic structure with well-defined and homogeneous features.

\section{Canon (Italian: 'canone')}

Texts constituting a 'canon' display the repetition of a style over time, i.e. canonical styles lose their original spontaneity and repeat themselves nearly unchanged over a period of several centuries, for extra-graphic, historical and cultural reasons. Even if a text within a canon has a unitary and closed graphic structure, it may also have an internal dynamic, with chronological and geographical differences. Moreover, when a canon comes into conflict with contemporary tastes and graphic techniques, it becomes difficult to maintain it in use, and for this reason the graphic rules are no longer respected: the shapes of individual letters are repeated, but not their structure (i.e. the number, sequence and direction of strokes), alongside the addition of extraneous elements, especially those with aesthetic functions.

\section{Normative script (Italian: 'scrittura normativa')}

In a recent publication Cavallo 2008, 15, has questioned the very concept of canons, since such terminology is 'too rigid for graphic forms which are lacking in any theoretical basis of fixed rules which need to be followed, and which also are not required models but simply represent one possible choice among others'; for this reason he now prefers the term 'normative scripts', meaning those scripts in which certain characteristics define a recognizable physiognomy for a certain (undefined) period, without however forcing them into the framework of a canon defined by fixed and immutable rules. 